\title{
Caractérisation par ellipsométrie spectroscopique de films minces de tellurure de bismuth obtenus par voie électrochimique
}

\author{
A. Zimmer ${ }^{1}$, N. Stein ${ }^{1}$, C. Boulanger ${ }^{1}$ et L. Johann ${ }^{2}$ \\ ${ }^{1}$ Laboratoire d'Électrochimie des Matériaux UMR CNRS 7555, Université de Metz, \\ 1 Boulevard Arago, CP 87811, 57078 Metz Cedex 3, France \\ ${ }^{2}$ Laboratoire de Physique des Liquides et des Interfaces, Université de Metz, \\ 1 Boulevard Arago, CP 87811, 57078 Metz Cedex 3, France
}

\begin{abstract}
Résumé. Des films de tellurure de bismuth $\left(\mathrm{Bi}_{2} \mathrm{Te}_{3}\right)$ d'épaisseur proche de $1 \mathrm{Pm}$ ont été développés par voie électrochimique. Leurs indices optiques ont été déterminés par ellipsométrie spectroscopique (SE). Le domaine spectral des indices optiques s'étend de $400 \mathrm{~nm}$ à $1300 \mathrm{~nm}$. L'ellipsométrie spectroscopique à angle d'incidence variable (VASE) a été utilisée pour corréler les données SE. Cette partie a été complétée par des analyses par microscopie à force atomique (AFM) qui ont permis de déterminer la rugosité des films. A partir de ces résultats et en associant l'absorption fondamentale des films de $\mathrm{Bi}_{2} \mathrm{Te}_{3}$ à une transition indirecte, l'énergie de bande interdite a été évaluée à $0,3 \mathrm{eV}$. Par ailleurs des mesures associant ellipsométrie spectroscopique à temps réel et électrochimie ont pu être réalisées. Ainsi les premiers instants de croissance ont été observés.
\end{abstract}

\section{INTRODUCTION}

Le tellurure de bismuth $\left(\mathrm{Bi}_{2} \mathrm{Te}_{3}\right)$ et ses dérivés ternaires $\left(\mathrm{Bi}_{2}(\mathrm{Te}, \mathrm{Se})_{3},(\mathrm{Bi}, \mathrm{Sb})_{2} \mathrm{Te}_{3}\right)$ sont considérés comme les meilleurs matériaux en vue d'applications thermoélectriques à température ambiante [1]. Différents protocoles de synthèses électrochimiques ont déjà été proposés conduisant à des films minces de composition et de stœchiométrie précises aux propriétés de transport et électroniques spécifiques [2,3]. Ce travail s'intègre dans une démarche fondamentale visant à caractériser les films obtenus ainsi qu'à déterminer leurs mécanismes de croissance. Les résultats présentés correspondent à une étude réalisée par ellipsométrie spectroscopique (SE). L'ellipsométrie est une technique optique d'analyse de surface non destructive permettant d'accéder aux propriétés optiques des films analysés. L'étude a été menée tout d'abord par des caractérisations ex-situ de films minces électrodéposés puis par des analyses in-situ au cours de procédés électrochimiques d'élaboration.

\section{PARTIE EXPERIMENTALE}

Le tellurure de bismuth est électrodéposé à partir d'une solution d'acide nitrique $1 \mathrm{M}$ contenant les espèces $\mathrm{Bi}^{3+}$ et $\mathrm{Te}^{\mathrm{IV}+}$ (concentration de $10^{-2} \mathrm{M}$ ) suivant la réaction :

$$
\mathrm{HTeO}_{2}^{+}+2 \mathrm{Bi}^{3+}+18 \mathrm{e}^{\square}+9 \mathrm{H}^{+} \mathrm{O} \quad \mathrm{Bi}_{2} \mathrm{Te}_{3}+6 \mathrm{H}_{2} \mathrm{O}
$$


Le composé stœchiométrique peut s'obtenir à potentiel imposé $(\mathrm{E}=\square 75 \mathrm{mV} / \mathrm{ECS})$ ou à intensité imposée $\left(j=\square 0,2 \mathrm{~A} / \mathrm{dm}^{2}\right)$ [4]. Un agent tensioactif a été ajouté pour diminuer la rugosité (gomme arabique en mode intensiostatique, dodécylsulfate de sodium en mode potentiostatique). L'étude a été menée dans un montage classique à trois électrodes utilisant une électrode au calomel à $\mathrm{KCl}$ saturé comme électrode de référence, une électrode auxiliaire en platine et une surface polie en or comme électrode de travail. Les films réalisés sont rincés successivement par de l'eau distillée, de l'acide nitrique $1 \mathrm{M}$ et du méthanol avant d'être séchés. Ils sont ensuite analysés par ellipsométrie spectroscopique (étude ex-situ).

L'ellipsométrie est une technique optique qui mesure le changement de l'état de polarisation d'une lumière polarisée après réflexion sur la surface de l'échantillon analysé. Les paramètres directement mesurables sont les angles ellipsométriques Psi $(<)$ et Delta (' $)$ définis par l'équation suivante :

$$
U \tan <e^{j^{\prime}} \quad \frac{R_{p}}{R_{s}}
$$

avec $U$ le rapport complexe des coefficients de réflexion de la lumière polarisée parallèlement $\left(R_{p}\right)$ et perpendiculairement $\left(\mathrm{R}_{\mathrm{s}}\right)$ au plan d'incidence [5]. L'ellipsométrie est une technique indirecte. Mis à part quelques cas, il faut en effet utiliser des méthodes numériques de minimalisation pour déterminer les grandeurs physiques. Celles-ci sont ajustées à partir de valeurs initiales de manière à diminuer l'écart entre les grandeurs ellipsométriques théoriques et expérimentales. Un algorithme de type Marquardt [6] est ainsi utilisé pour minimiser une fonction d'écart notée $\mathrm{F}^{2}$ [7].

Les mesures ellipsométriques ont été effectuées au sein du groupe de Spectrométrie des Interfaces du Laboratoire de Physique des Liquides et des Interfaces de l'Université de Metz (LPLI). La source lumineuse est constituée d'une lampe Xénon (puissance de $70 \mathrm{~W}$ ). Le domaine spectral analysé correspond au visible et au proche infrarouge jusqu'à $1300 \mathrm{~nm}$. La morphologie de surface des films a été analysée par microscopie à force atomique (AFM) en mode contact (Topometrix Explorer Ecu+ TMX 1010). La stœchiométrie des films a été contrôlée par Spectrométrie de Fluorescence X (Bruker S4 Explorer).

Nous avons réalisé des films en mode intensiostatique pour l'étude ex-situ et en mode potentiostatique pour l'étude in-situ afin d'obtenir des épaisseurs voisines de $1 \mathrm{Pm}$.

\section{RÉSULTATS ET DISCUSSION}

\section{1 Étude ellipsométrique $e x$-situ}

La stœchiométrie d'un des films obtenus vaut $\mathrm{Bi}_{1,93} \mathrm{Te}_{3,07}(\mathrm{r} 0,05)$. Des mesures ellipsométriques ont été réalisées sur cinq échantillons à trois angles d'incidence $\left(65^{\circ}, 70^{\circ}, 75^{\circ}\right)$. Une bonne reproductibilité des mesures est observée. Les indices optiques présentés dans cet article correspondent à la moyenne de l'ensemble de ces données.

\subsubsection{Spectres ellipsométriques}

La figure 1 (points) présente les spectres ellipsométriques expérimentaux. La dispersion des points expérimentaux au-delà de 1000 nm correspond à la moindre sensibilité du détecteur utilisé. 

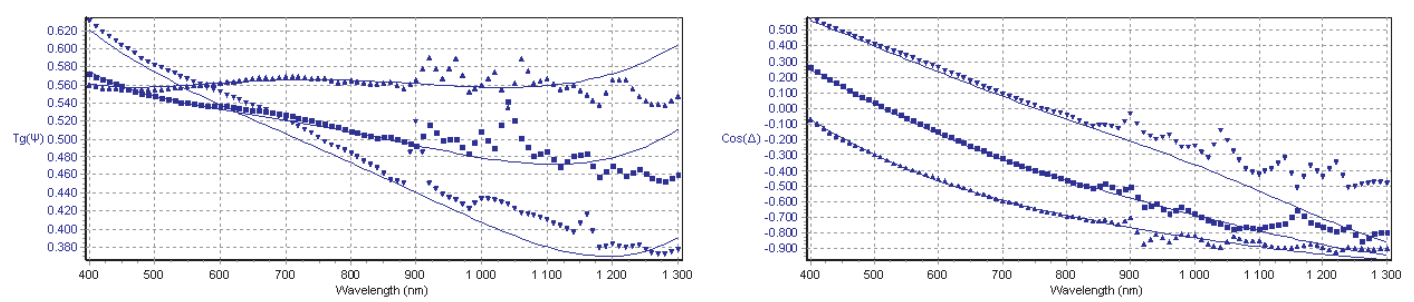

Figure 1. Données ellipsométriques expérimentales (points) et modélisées (traits) : tan < (à gauche) et $\cos ^{\prime}$ (à droite). Angle d'incidence : $65^{\circ}(\boldsymbol{\Delta}), 70^{\circ}(\boldsymbol{\nabla}), 75^{\circ}(\boldsymbol{\nabla})$.

\subsubsection{Présentation du modèle optique}

Afin d'accéder aux grandeurs physiques, il est nécessaire d'établir un modèle décrivant les interfaces substrat | film | milieu ambiant. Le modèle retenu correspond à un substrat massif $\mathrm{de} \mathrm{Bi}_{2} \mathrm{Te}_{3}$ comportant une couche surfacique rugueuse (figure 2). La couche est composée d'un mélange de $\mathrm{Bi}_{2} \mathrm{Te}_{3}$ et de milieu ambiant suivant la théorie du milieu effectif de Bruggeman (BEMA) [8]. Cette couche est caractérisée par une épaisseur $\mathrm{d}^{\mathrm{SE}}$ et une fraction volumique $\mathrm{f}$ en matériau. Le film a été considéré comme optiquement isotrope. Grâce à ce modèle les indices optiques ont pu être déterminés ainsi que la rugosité des films. Pour décrire les indices de réfraction (n) et d'extinction (k), nous avons choisi une loi de dispersion dérivée de la loi de Sellmeier (étendue à l'absorption) suivant les équations indiquées à la figure 2 .

Milieu ambiant

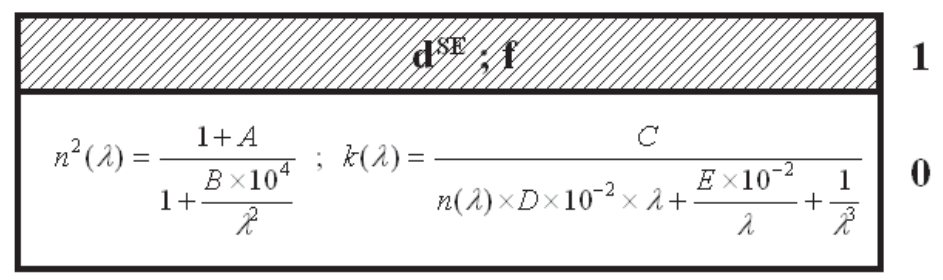

Figure 2. Modèle à 7 paramètres : indices optiques (n, k) du substrat (0) décrit par la loi de Sellmeier étendue (paramètres d'ajustement $\mathrm{A}$ à $\mathrm{E}$, Ola longueur d'onde en $\mu \mathrm{m}$ ) et couche surfacique (1) d'épaisseur $\mathrm{d}^{\mathrm{SE}}$ constituée d'un mélange de substrat (de fraction volumique f) et de milieu ambiant.

\subsubsection{Indices optiques - Rugosité}

La figure 1 (traits) présente les spectres ellipsométriques simulés sur l'ensemble du domaine après ajustement des paramètres du modèle sur le domaine du visible étendu à $900 \mathrm{~nm}$. Un très bon accord est observé entre les valeurs expérimentales et les valeurs simulées jusqu'à $1300 \mathrm{~nm}$. Le tableau 1 rassemble les valeurs des paramètres du modèle pour le meilleur ajustement obtenu $\left(\mathrm{F}^{2}\right.$ minimum). La figure 3 présente les indices résultants de cet ajustement. Les indices ainsi obtenus ont été comparés avec ceux obtenus par Cui et al. [9] par MOCVD (metalorganic chemical vapor deposition). Nous constatons une différence entre les données qui peut s'expliquer par la morphologie des films suivant la méthode de synthèse utilisée. Ces indices constituent un premier résultat. Le but fondamental étant de pouvoir lier les indices optiques avec la stœchiométrie des films, des analyses sont prévues pour d'autres stœchiométries de $\mathrm{Bi}_{2} \mathrm{Te}_{3}$. 
Tableau 1. Valeurs des paramètres du modèle (figure 2) après ajustement.

\begin{tabular}{|c|c|c|c|c|c|c|c|c|}
\hline Paramètre & A & B & C & D & E & $\mathrm{d}^{\mathrm{SE}}(\AA)$ & $\mathrm{f}(\%)$ & $\mathrm{F}^{2}$ \\
\hline Valeur & 25 r 4 & -258 r 32 & 200 r 27 & 0,56 r 0,09 & 430 r 59 & 118 r 17 & 10,6 r 1,6 & 0,557 \\
\hline
\end{tabular}

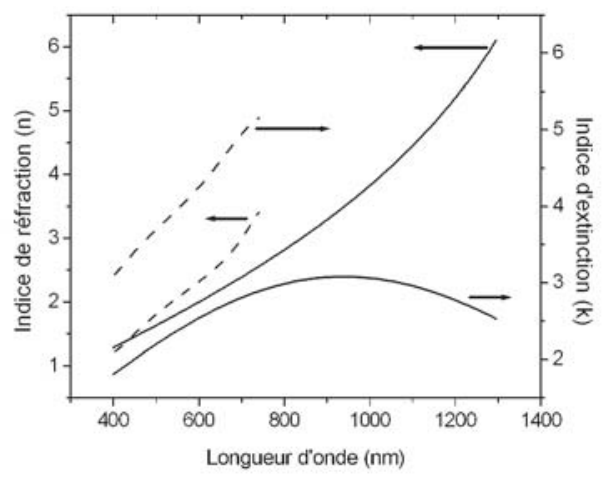

Figure 3. Indices optiques de $\mathrm{Bi}_{2} \mathrm{Te}_{3}$ déposé par Figure 4. Image $\mathrm{AFM} \mathrm{de} \mathrm{Bi}_{2} \mathrm{Te}_{3}$ (mode contact).

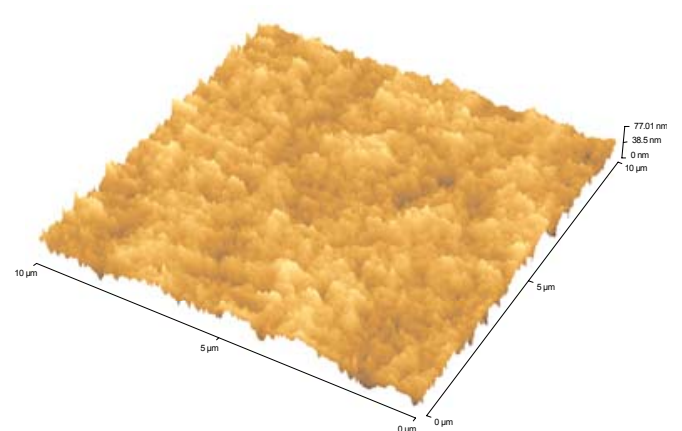
électrochimie $(\square)$ et d'après Cui et al.[9] (----).

Des analyses ont été menées par microscopie à force atomique (AFM) sur les films minces de tellurure de bismuth afin de corréler les valeurs de rugosités. Les surfaces analysées correspondent à

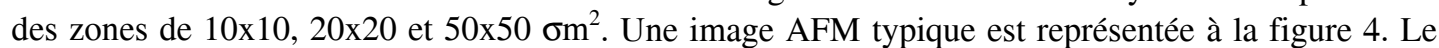
tableau 2 présente les valeurs de rugosités déterminées à partir de la figure 4. Ces valeurs correspondent au critère statistique RMS (root mean square, noté ici $\mathrm{d}^{\mathrm{RMS}}$ ) représentant l'écart quadratique moyen du profil analysé. Les valeurs obtenues selon les surfaces analysées sont relativement constantes ; la moyenne est de $94,4 \AA$. Le rapport $\mathrm{d}^{\mathrm{SE}} / \mathrm{d}^{\mathrm{RMS}}$ vaut, en moyenne, 1,25 ce qui est cohérent avec la littérature [10].

Tableau 2. Rugosités obtenues par analyse AFM.

\begin{tabular}{|c|c|c|c|c|}
\hline Zone $\left(\mathrm{Pm}^{2}\right)$ & 100 & 400 & 2500 & Moyenne \\
\hline $\mathrm{d}^{\mathrm{RMS}}[\AA]$ & 98,3 & 95,9 & 88,9 & 94,4 \\
\hline $\mathrm{d}^{\mathrm{SE}} / \mathrm{d}^{\mathrm{RMS}}$ & $1,20 \mathrm{r} 0,17$ & $1,23 \mathrm{r} 0,18$ & $1,33 \mathrm{r} 0,19$ & $1,25 \mathrm{r} 0,18$ \\
\hline
\end{tabular}

\subsubsection{Détermination du gap du semi-conducteur}

Comme $\mathrm{Bi}_{2} \mathrm{Te}_{3}$ est généralement considéré comme un semi-conducteur à gap indirect $[11,12]$, le coefficient d'absorption $\mathrm{D}\left[\mathrm{l}=4 \mathrm{Sk} / \mathrm{O}\right.$ ), l'énergie de bande interdite $\mathrm{E}_{\mathrm{g}}$ (gap) et l'énergie $\mathrm{E}$ sont liés par la relation $\mathrm{DE}=\mathrm{A}\left(\mathrm{E} \square \mathrm{E}_{\mathrm{g}}\right)^{2}$ où $\mathrm{A}$ est une constante. Le gap peut ainsi être évalué en extrapolant la portion linéaire du graphique (DE) ${ }^{1 / 2}$ en fonction de l'énergie $\mathrm{E}$ (figure 5). 


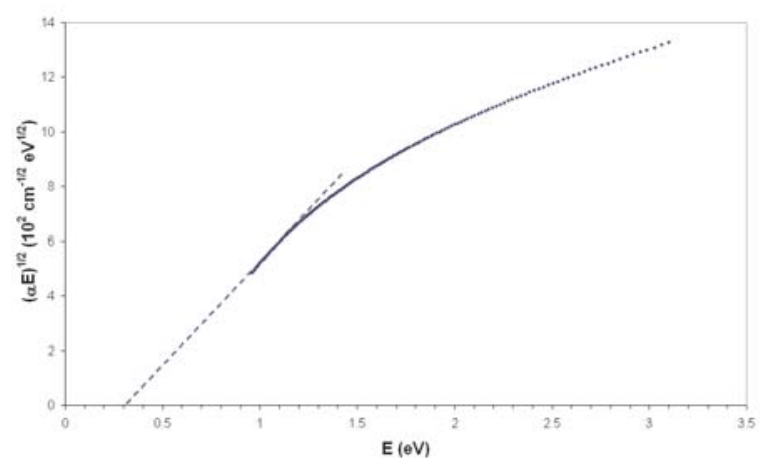

Figure 5. Tracé de $(\mathrm{DE})^{1 / 2}$ en fonction de l'énergie $\mathrm{E}$ pour déterminer le gap optique de $\mathrm{Bi}_{2} \mathrm{Te}_{3}$ par extrapolation de la partie linéaire de la courbe.

La valeur obtenue de $0,3 \mathrm{eV}$ est en accord avec celles de la littérature $(0,11 \square 0,3 \mathrm{eV})$ [11-13] pour des conditions de synthèse différentes. A terme, des mesures dans l'IR permettront d'affiner cette valeur.

\section{2 Étude ellipsométrique in-situ}

A l'aide d'une cellule de couplage (avec un angle d'incidence fixe de $66^{\circ}$ ) permettant la mesure des angles ellipsométriques pendant l'électrodéposition des films, des premières mesures associant ellipsométrie spectroscopique à temps réel et électrochimie ont pu être réalisées. Les premiers instants de croissance ont été observés (figure 6). Deux zones sont mises en évidence. Jusqu'à environ $250 \mathrm{~s}$, une zone de forte variations des angles ellipsométriques est observée. Cet intervalle est cohérent avec le fait que les mesures sont influencées par le substrat d'or pour les faibles épaisseurs (recouvrement partiel du substrat par le film). Cette influence du substrat peut être évaluée par la loi de Beer [14] à travers le rapport $1 \square_{\square}$ (figure 7). Au-delà de $250 \mathrm{~s}$, l'évolution relativement faible des signaux optiques correspond probablement à l'évolution de l'état de surface du film.
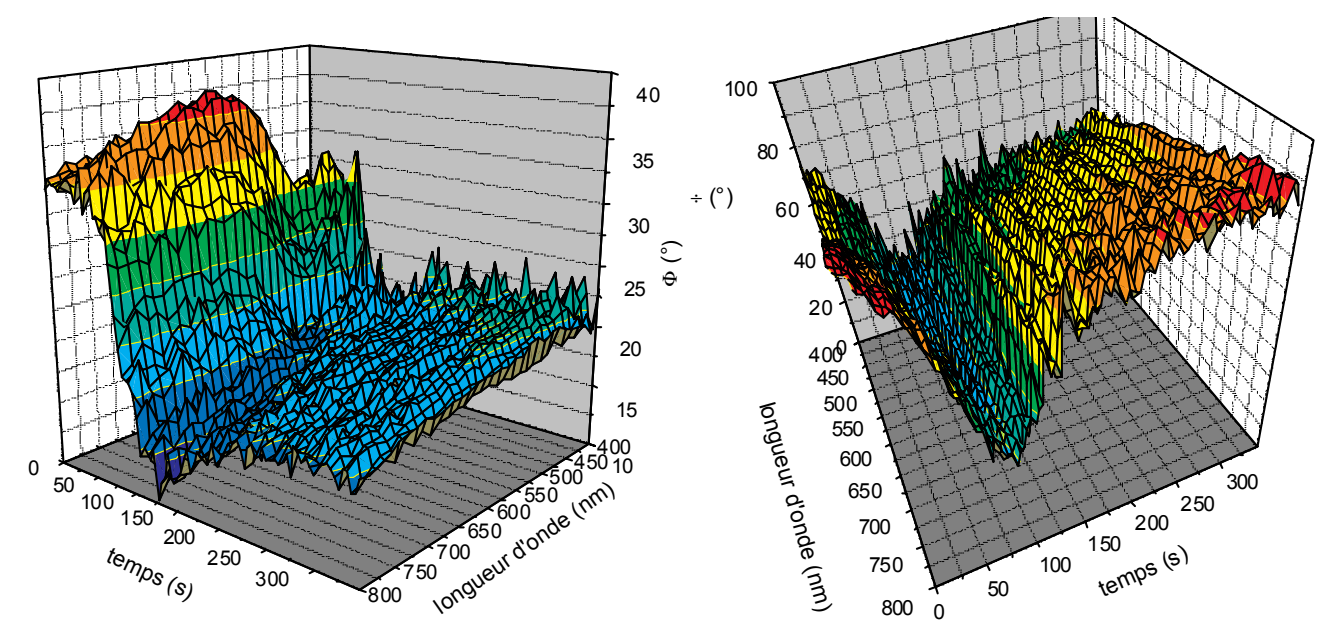

Figure 6. Angles ellipsométriques < \à gauche) et ' Џà droite) en fonction de la longueur d'onde et du temps au cours de la croissance d'un film de $\mathrm{Bi}_{2} \mathrm{Te}_{3}$ en mode potentiostatique ( $\left.\mathrm{E}=\square 75 \mathrm{mV} / \mathrm{ECS}\right)$. 


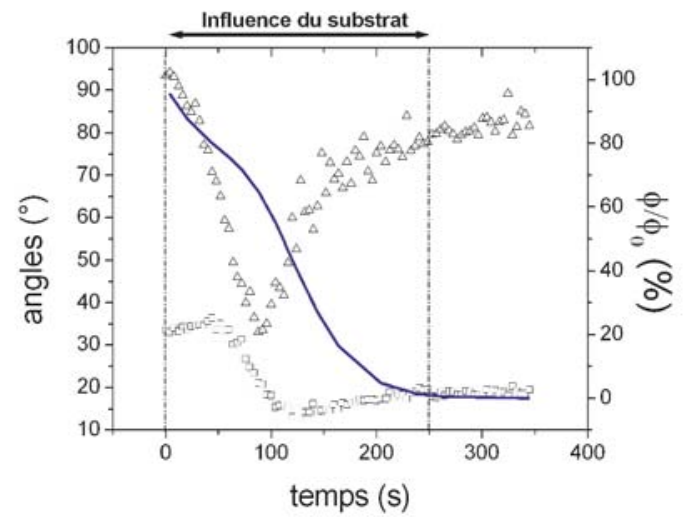

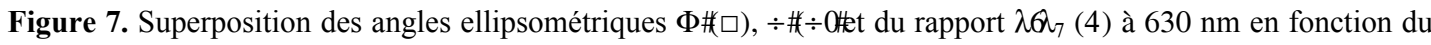
temps au cours de la croissance d'un film de $\mathrm{Bi}_{2} \mathrm{Te}_{3}$.

\section{CONCLUSION}

Sur le plan fondamental, les indices optiques du tellurure de bismuth obtenus par électrochimie ont été déterminés par SE sur le domaine 400-1300 nm pour une composition proche de la stœchiométrie. Le gap a par ailleurs été estimé à $0,3 \mathrm{eV}$. Concernant le suivi in-situ, les premiers instants ont été suivi avec succès avec le nouveau montage couplant électrochimie et ellipsométrie. Ces premiers instants semblent répondrent à un mécanisme complexe. Le recours à des mesures combinant microbalance à quartz électrochimique (EQCM, Electrochemical Quartz Crystal Microbalance) et ellipsométrie temps réel permettra d'affiner la compréhension des phénomènes mis en jeu.

\section{Références}

[1] G.S. Nolas, J. Sharp and H.J. Goldsmid, Thermoelectrics Basic Principles and New Materials Developments (Springer, New-York, 2001) pp. 126-130.

[2] P. Magri, C. Boulanger, J.M. Lecuire, J. Mater. Chem. 6 (1996) 773-779.

[3] S. Michel, N. Stein, M. Schneider, C. Boulanger, J.M. Lecuire, J. Appl. Electrochem., 33 (2003) 23-27.

[4] S. Michel, Contribution à l'élaboration d'électrodépôts de matériaux thermoélectriques de type $\mathrm{Bi}_{2} \mathrm{Te}_{3}$ et $\mathrm{Bi}_{2}\left(\mathrm{Te}_{0,9} \mathrm{Se}_{0,1}\right)_{3}$ (Thèse de l'Université de Metz, 2003) pp. 57, 86.

[5] R.M.A. Azzam and N.M. Bashara, Ellipsometry and Polarized Light (North-Holland, Amsterdam, 1977) chap.4.

[6] D.W. Marquardt, J. Soc. Ind. Apll. Math. 11 (1963) 431.

[7] Software DELTAPSI 2, Jobin Yvon S.A.S., Longjumeau, France (www.jobinyvon.com).

[8] D.G.A. Bruggeman, Ann. Phys. 24 (1935) 636.

[9] H.Cui, I.B. Bhat, B. O’Quinn and R. Venkatasubramanian, J. Electron. Mater., 28 (1999) 11111114.

[10] H.Fujiwara, J.Koh, Pirovira and R.W.Collins, Phys.Rev.B, 61 (2000) 10832-10844.

[11] I.G. Austin, Proc. of the Phys. Society, 72 (1958) 545-552.

[12] El H. Kaddouri, T. Maurice, X. Gratens, S. Charar, S. Benet, A. Mefleh, J.C. Tedenac and B. Liautard, Phys. Stat. Sol. (a) 176 (1999) 1071-1076.

[13] S.K. Mishra, S.Satpahthy and O.Jepsen, J. Phys.: Condens. Matter 9 (1997) 461-470.

[14] H. Kuzmany, Solid-State Spectroscopy, An Introduction (Springer, Berlin, 1998) pp.102-103. 